\title{
Anticrossing of Longitudinal and Transverse Modes in Simple Fluids (Supporting Information)
}

\author{
Nikita P. Kryuchkov ${ }^{1}$, Vadim V. Brazhkin ${ }^{2}$, and Stanislav O. Yurchenko ${ }^{1}$ \\ ${ }^{1}$ Bauman Moscow State Technical University, 2nd Baumanskaya street 5, 105005 Moscow, Russia \\ ${ }^{2}$ Institute for High Pressure Physics RAS, Kaluzhskoe shosse, 14, Troitsk, Moscow, 108840 Russia
}

Pseudoharmonic approximation. The Hamiltonian of a system of identical particles is

$$
H=\sum_{s} \frac{m \mathbf{v}_{s}^{2}}{2}+U\left(\mathbf{r}_{1}, \mathbf{r}_{2}, \ldots \mathbf{r}_{N}\right)
$$

where $m$ is the particle mass (which we assume equal to unity), $\mathbf{v}_{s}=\dot{\mathbf{r}}_{s}$ is the velocity of the $s$-th particle, $U$ is the potential energy of the system, and summation is performed over all particles in the system.

In the case of plane waves propagating along the system, we have

$$
\mathbf{r}_{s}(t)-\mathbf{r}_{s 0}=\sum_{\mathbf{q}} \mathbf{a}_{\mathbf{q}}(t) e^{i \mathbf{q} \mathbf{r}_{s 0}}=\sum_{\mathbf{q}} \mathbf{A}_{\mathbf{q}}(t) \cos \left(\mathbf{q} \mathbf{r}_{s 0}+\psi_{\mathbf{q}}\right),
$$

where $\mathbf{r}_{s 0}=\mathbf{r}_{s}(0)$ is the particle position at the initial time $t=0 ; \mathbf{a}_{\mathbf{q}}$ and $\mathbf{A}_{\mathbf{q}}$ are the complex and vector amplitudes of $q$-fluctuation, respectively $\left(\left|\mathbf{a}_{\mathbf{q}}\right|=\left|\mathbf{A}_{\mathbf{q}}\right|\right)$; and $\psi_{\mathbf{q}}$ is the phase. The velocities of particles are $\mathbf{v}_{s}(t)=$ $\sum_{\mathbf{q}} \dot{\mathbf{a}}_{\mathbf{q}} e^{i \mathbf{q} \mathbf{q}_{s 0}}$ and, hence, $\sum_{s} \mathbf{v}_{s}^{2}=\sum_{\mathbf{q}}\left|\dot{\mathbf{a}}_{\mathbf{q}}\right|^{2}=\sum_{\mathbf{q}} \dot{A}_{\mathbf{q}}^{2}$.

In the pseudoharmonic approximation, we assume that the phases $\psi_{\mathbf{q}}$ (independent for different $\mathbf{q}$ ) are constant, the fluctuations are described by the set of amplitudes $\mathbf{A}_{\mathbf{q}}$, the Hamiltonian of the system is the sum of terms corresponding to different $\mathbf{q}$-harmonics, while damping and generation of excitations (due to their interactions with each other) are described using the dissipationfluctuation theorem, as explained in the Letter.

Equation for eigenfrequencies in the 3D case. In this case, the pseudoharmonic Hamiltonian can be written in the form ( $q$ indices are omitted)

$$
\begin{aligned}
H= & \frac{1}{2}\left(\dot{A}_{\|}^{2}+\dot{A}_{\perp}^{2}+\dot{A}_{\perp}^{\prime 2}\right)+\epsilon \Omega_{\|} \Omega_{\perp} A_{\|}\left(A_{\perp}+A_{\perp}^{\prime}\right) \\
& +\frac{1}{2}\left(\Omega_{\|}^{2} A_{\|}^{2}+\Omega_{\perp}^{2} A_{\perp}^{2}+\Omega_{\perp}^{2} A_{\perp}^{\prime 2}\right),
\end{aligned}
$$

where $A_{\perp}$ and $A_{\perp}^{\prime}$ are the amplitudes of fluctuations in two perpendicular transverse directions. Due to the isotropy of simple fluids and, in particular, equivalence of properties in all transverse directions, corresponding frequencies, coupling constant, and damping rates of the modes are the same for the both transverse directions. However, there is no term $\propto A_{\perp} A_{\perp}^{\prime}$ in Eq. (S3); otherwise, it would lead to artificial splitting of eigenfrequencies of transverse modes and broken symmetry between them.

The dynamic equations for amplitudes can be derived in the same manner as explained in the main text:

$$
\begin{aligned}
& \ddot{A}_{\|}+2 \Gamma_{\|} \dot{A}_{\|}+\Omega_{\|}^{2} A_{\|}+\epsilon \Omega_{\|} \Omega_{\perp}\left(A_{\perp}+A_{\perp}^{\prime}\right)=\xi_{\|}(t), \\
& \ddot{A}_{\perp}+2 \Gamma_{\perp} \dot{A}_{\perp}+\Omega_{\perp}^{2} A_{\perp}+\epsilon \Omega_{\|} \Omega_{\perp} A_{\|}=\xi_{\perp}(t), \\
& \ddot{A}_{\perp}^{\prime}+2 \Gamma_{\perp} \dot{A}_{\perp}^{\prime}+\Omega_{\perp}^{2} A_{\perp}^{\prime}+\epsilon \Omega_{\|} \Omega_{\perp} A_{\|}=\xi_{\perp}^{\prime}(t),
\end{aligned}
$$

where $\xi_{\perp}^{\prime}(t)$ is the fluctuation "force" for the second transverse direction. Analysis of the system (S4) yields the same equation (4) for eigenfrequencies as in the $2 \mathrm{D}$ case.

Suitability of analysis of dispersion relation with $C(q, \omega)$-maxima. The method based on analysis of $C(q, \omega)$-maxima is widely used, but it yields appropriate results for $\omega(q)$ (without any information about damping rate of the excitations) only if $C(q, \omega)$ has narrow peaks in $(q, \omega)$ plane. Typically, this corresponds to a case of crystals at low temperatures, as long as anharmonicity effects are weak.

In fluids, the situation is opposite due to developed anharmonicity and disordered structure, which lead to significant broadening of the peaks, related to decrease of excitation lifetime (inverse damping rate) and significant overlap of the longitudinal and transverse components of $C(q, \omega)$ in $(q, \omega)$ plane. In result, the method, based on analysis of $C(q, \omega)$-maxima, becomes unsuitable in fluids. This statement follows from simple analysis of Eq. (7) in the main text, from where one can readily obtain the following expression for the frequency $\omega_{m}$ of $C(q, \omega)$-maxima:

$$
\omega_{m}=\omega_{\perp} \operatorname{Re}\left[2 \sqrt{1+\left(\frac{\Gamma_{\perp}}{\omega_{\perp}}\right)^{2}}-\left(\frac{\Gamma_{\perp}}{\omega_{\perp}}\right)^{2}-1\right]^{1 / 2} .
$$

We see in Eq. (S5) that $\omega_{m} \simeq \omega_{\perp}$ only if $\Gamma_{\perp} / \omega_{\perp} \ll 1$, while, in fluids, $\Gamma_{\perp}$ and $\omega_{\perp}$ are typically comparable with each other (or even $\Gamma_{\perp} / \omega_{\perp} \gg 1$ ). Moreover, with increase in $\Gamma_{\perp} / \omega_{\perp}$, the frequency $\omega_{m}$ drops and gets equal to zero at $\Gamma_{\perp} / \omega_{\perp}=\sqrt{3}$, even if $\omega_{\perp} \neq$ 0 . Note that, for low-frequency (transverse) branch of excitations, $\Gamma_{\perp} / \omega_{\perp} \gg 1$ at small (q-gap) and large wavenumbers. [1]

[1] N. P. Kryuchkov, L. A. Mistryukova, V. V. Brazhkin, and S. O. Yurchenko, Scientific Reports 9, 10483 (2019) 

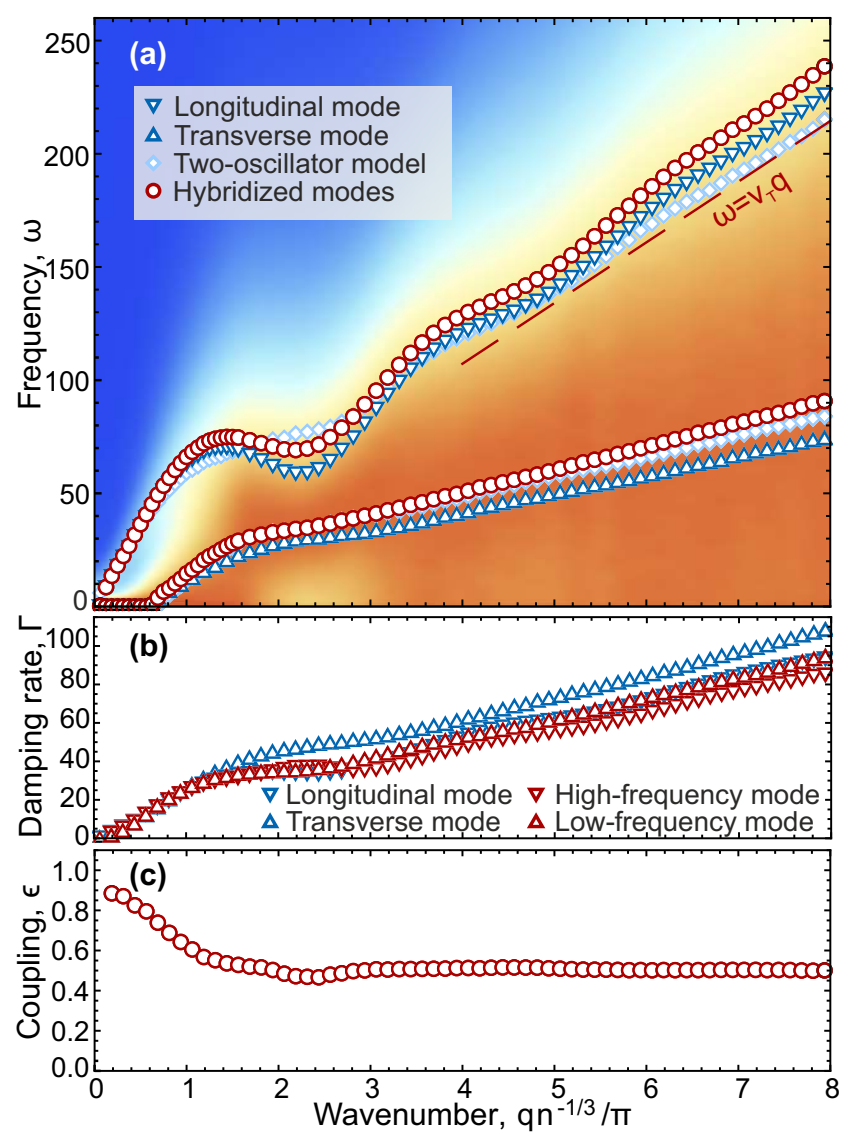

Figure S1. Anticrossing of modes in noble gas fluids at high temperatures: Spectra of collective excitations measured using different approaches at a temperature of 36.4 (in dimensionless units). (a) Oscillation frequencies obtained by different ways, (b) damping rates, (c) coupling constant for excitations with different polarizations. Details of description are the same as in Fig. 1 in the main text. 

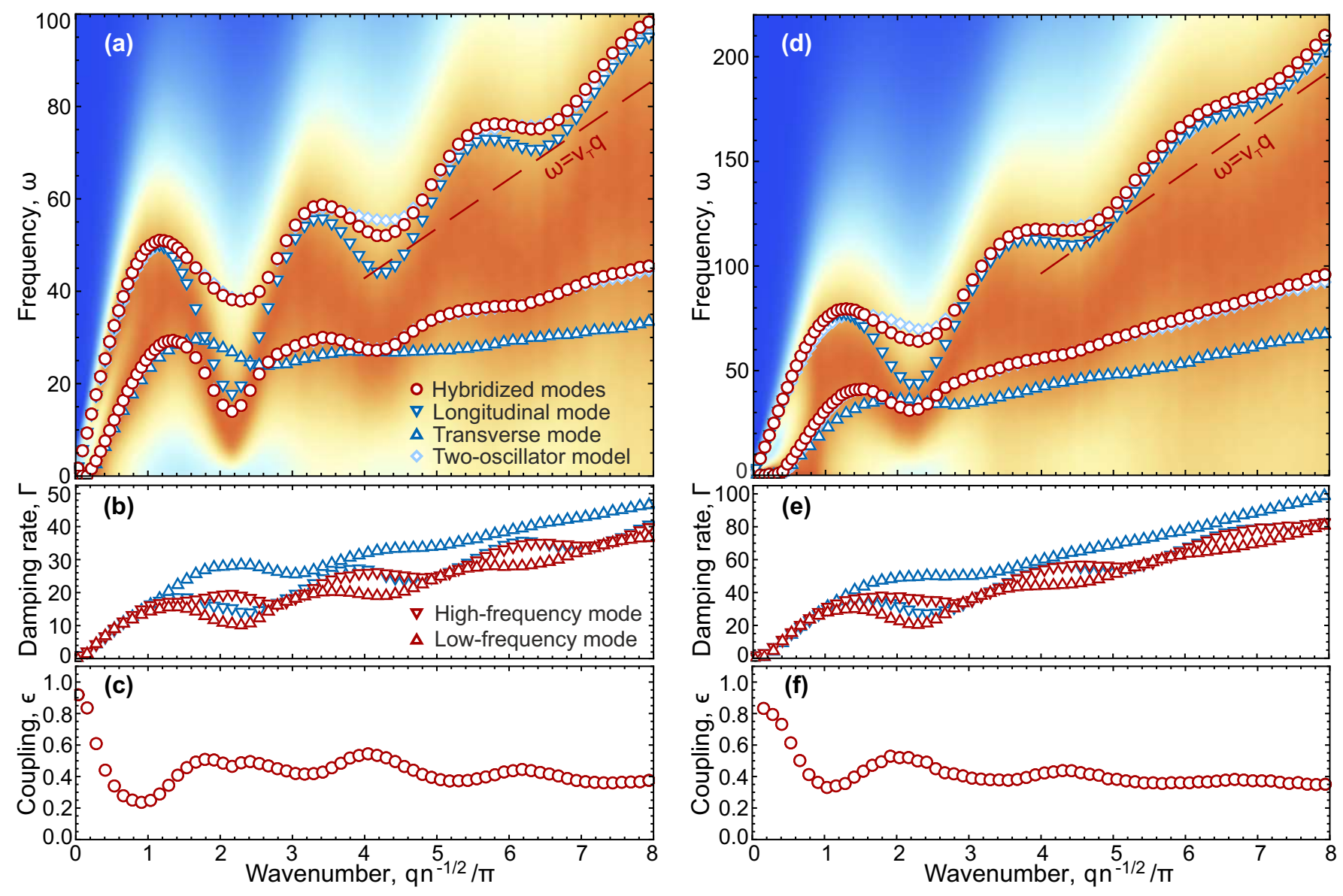

Figure S2. Anticrossing of longitudinal and transverse modes in 2D Lennard-Jones fluids: Spectra of collective excitations measured using different approaches at the dimensionless temperatures $T=5.8(\mathrm{a}-\mathrm{c})$ and $T=29.5(\mathrm{~d}-\mathrm{f})$. (a, d) Frequencies of collective excitations obtained by different ways, (b, e) damping rates, (c, f) coupling constant. Details of description are the same as in Fig. 1 in the main text. 